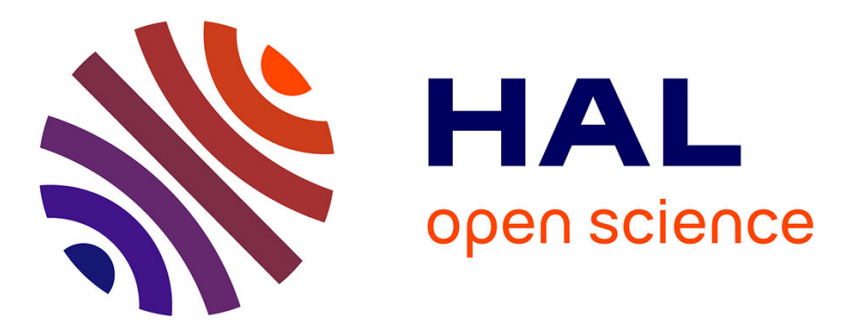

\title{
First-principles study of the atomic structure of glassy Ga10Ge15Te75
}

Z. Chaker, G. Ori, M. Boero, C. Massobrio, E. Furet, A. Bouzid

\section{To cite this version:}

Z. Chaker, G. Ori, M. Boero, C. Massobrio, E. Furet, et al.. First-principles study of the atomic structure of glassy Ga10Ge15Te75. Journal of Non-Crystalline Solids, 2015, 498, pp.338-344. 10.1016/j.jnoncrysol.2018.03.039 . hal-01861419

HAL Id: hal-01861419

\section{https://hal-univ-rennes1.archives-ouvertes.fr/hal-01861419}

Submitted on 7 Nov 2018

HAL is a multi-disciplinary open access archive for the deposit and dissemination of scientific research documents, whether they are published or not. The documents may come from teaching and research institutions in France or abroad, or from public or private research centers.
L'archive ouverte pluridisciplinaire HAL, est destinée au dépôt et à la diffusion de documents scientifiques de niveau recherche, publiés ou non, émanant des établissements d'enseignement et de recherche français ou étrangers, des laboratoires publics ou privés. 


\title{
First-principles study of the atomic structure of glassy $\mathrm{Ga}_{10} \mathrm{Ge}_{15} \mathrm{Te}_{75}$
}

\author{
Z. Chaker, G. Ori, M. Boero, C. Massobrio \\ Institut de Physique et Chimie des Matériaux de Strasbourg, Université de Strasbourg, \\ CNRS UMR 7504, F-67034 STRASBOURG Cedex 2, France \\ E. Furet \\ Institut des Sciences Chimiques de Rennes UMR 6226, École Nationale Supérieure de Chimie de Rennes, \\ Avenue du Général Leclerc, CS 50837, F-35708 Rennes, France \\ A. Bouzid \\ Chaire de Simulation à l'Échelle Atomique (CSEA), École Polytechnique Fédérale de Lausanne (EPFL), \\ CH-1015 Lausanne, Switzerland
}

\begin{abstract}
First-principles molecular dynamics have been employed to highlight the structural properties of glassy $\mathrm{Ga}_{10} \mathrm{Ge}_{15} \mathrm{Te}_{75}$ (GGT), a promising disordered system for infrared applications. Our approach relies entirely on the predictive power of density functional theory, in combination with a careful choice of the exchangecorrelation functional and the account of van der Waals interactions. Glassy GGT can be viewed as a predominantly tetrahedral network, both with respect to Ge and Ga atoms. Te atoms are mostly twofold coordinated to all combinations of pairs of $\mathrm{Ge}, \mathrm{Ga}$ or Te atoms. We find little evidence of intermediate range order in the partial structure factors.
\end{abstract}

Key words: Disordered materials; Glassy chalcogenides; First-principles molecular dynamics; Density functional theory

\section{Introduction}

In the search of glassy stable materials having remarkably larger infrared (IR) transparency windows, Te-rich ternary compounds are well established for being highly performing and well adapted to various applications [1]. To build a technologically valuable Te-based ternary glass, Ge is an appropriate choice. This 5 is due to the existence of well characterized Ge-Te binary materials, notwithstanding a reduced glass forming range compared to the Ge-Se case. Focusing on compositions close to $\mathrm{GeTe}_{4}$, the inclusion of small amounts of Se, Ga or I was found to contribute to improved glass formation and stability against crystallization, thereby meeting the requirements of enhanced stability combined to exceptionally broad (up to $28 \mu \mathrm{m}$ ) IR windows $[1,2,3]$. It has to be reminded that, although somewhat similar in terms of the chemical compositions, this family of glasses is distinct from the phase change materials (PCM) glasses, for which the occurrence of 
rapid structural changes from the crystalline to the disordered phase is an essential prerequisite [4]. We have undertaken in recent years a major research effort devoted to elucidating the atomic structure of ternary Te-rich glasses via first-principles molecular dynamics (FPMD) simulations [5]. Our final goal is to provide a comprehensive picture of the glassy behavior (including structural, vibrational and electronic properties) based on a quantitative theoretical methodology with no a priori hypotheses on the nature of chemical bonding. In this paper, we describe the topology and the coordination network of glassy $\mathrm{Ga}_{10} \mathrm{Ge}_{15} \mathrm{Te}_{75}$ (also termed GGT hereafter) in the framework of density functional theory (DFT) Car-Parrinello FPMD. Our results point toward the existence of an extended tetrahedral network, in which Ga and Ge atoms are predominantly fourfold coordinated while Te atoms are mostly twofold or threefold coordinated. In addition, because of their concentration well above the stoichiometric one (i.e. Te twofold coordinated to either Ge or Ga fourfold coordinated) Te atoms are also found in homopolar Te-Te bonds. Throughout the paper we shall compare our findings with relevant experiments [6] and with DFT modeling results obtained by Voleska and coworkers [7] for a GaGeTe system of a slightly different concentration $\left(\mathrm{Ga}_{11} \mathrm{Ge}_{11} \mathrm{Te}_{78}\right)$. It is worth underlying that in Ref. [7], the DFT-FPMD approach was used in combination with structural refinement techniques such as reverse Monte Carlo (RMC-FPMD-PBE). Here we demonstrate that the high predictive power of DFT-FPMD, used in conjunction with a judicious choice of the exchange-correlation (XC) functional and a treatment of the van der Waals (vdW) interaction, allows achieving quantitative agreement with available structural data. This is obtained without any structural information issued from experiments. Our paper is organized as follows. In the first section, we describe our computational methods and models by pointing out specific choices for the exchange-correlation functionals and the dispersion (van der Waals) contributions. Then, the section devoted to the results contains our analysis of the total and partial structure factors, pair correlation functions, coordination numbers at various levels and, as final information, the local order parameter. The paper ends with some conclusive remarks.

\section{Computational methods and models}

Our simulations were performed within the Car-Parrinello [8] molecular dynamics (CPMD) method as implemented in the CPMD code [9]. We employ the BLYP exchange-correlation scheme due to Becke [10] (exchange part) and to Lee, Yang and Parr [11] (correlation part). This generalized gradient approximation (GGA) has an extended record of reliability for chalcogenide glasses, since it enhances the localized behavior of the electron density at the expenses of electronic delocalization effects that favor the metallic character ([13]) in reproducing the available experimental quantities, yielding a prominently tetrahedral atomic-scale network in which tetrahedral and defective octahedral motifs do coexist. Differences between BLYP and 
PBE atomic structures are less severe in the case of the PCM glass $\mathrm{Ge}_{2} \mathrm{Sb}_{2} \mathrm{Te}_{5}$ ([5]). For the core-valence interactions, we use a norm-conserving pseudo-potential according to the description of Troullier and Martins [14]. Nonlinear core corrections (NLCC) are adopted for Ga atoms according to the prescription of Louie [15]. In our calculations, a plane-wave basis set is chosen for the representation of the valence electrons with a corresponding energy cutoff of $40 \mathrm{Ry}$ and a restriction to the $\Gamma$ point for the Brillouin zone integration. This choice is substantiated by previous investigations on disordered chalcogenides $[2,16,17,18,19,20]$.

Long-range dispersion forces were accounted for according to the DFT-D2 formula proposed by Grimme [21]. Such a vdW correction is a thorough DFT-based formulation in which parameters are self-consistently tuned on different functionals, including those used (BLYP) or referred to (PBE) in this work. No experimental parameters are included in the construction of the specific $\mathrm{vdW}$ corrections and their inclusion does not affect at any stage the Kohn-Sham equations [22, 23], thus preserving the first-principles character of the electronic structure calculations.

The temperature of the ions was controlled via a Nosé-Hoover thermostat [24, 25]. We use the scheme proposed by Blöchl and Parrinello [26] to control the evolution of the temperature of the fictitious electronic degrees of freedom. The integration step is 5 a.u ( $0.12 \mathrm{fs})$, ensuring an optimal control of the conserved quantities all along the simulations.

Our GGT system is a periodic structure with a cubic cell containing 480 atoms (48 Ga, $72 \mathrm{Ge}, 360 \mathrm{Te}$ ) and a side equal to $25.65 \AA$. This composition is very close to the one studied by Voleská et al. [7]. Two models of the glassy $\mathrm{Ga}_{10} \mathrm{Ge}_{15} \mathrm{Te}_{75}$ were considered. To begin our investigation, we selected two sets of 480 coordinates taken from previous FPMD studies carried out on binary chalcogenide systems. For the first, the initial coordinates were taken from a previous sample of glassy $\mathrm{GeSe}_{2}$ ([12]). For the second, the initial coordinates were taken from glassy $\mathrm{GeSe}_{4}[16]$ ). As a reaction to the change in the identity of the chemical species, the system rearranges abruptly as manifested by the average lengths covered by the atoms (at least two interatomic distances) on a time period of a few ps. During this period, the system temperature can attain temperatures as high as $1000 \mathrm{~K}$ with transient diffusion coefficients of the order of $10^{-4} \mathrm{~cm}^{2} / \mathrm{s}$. Typically, such a non-equilibrium trajectory is already sufficient to lose memory of the initial configuration. After quenching to $\mathrm{T}=300 \mathrm{~K}$, the thermal cycles go as follows. In the first case, the trajectories produced encompass 5 ps at $\mathrm{T}=300 \mathrm{~K}, 4.5 \mathrm{ps}$ at $\mathrm{T}=600 \mathrm{~K}, 10 \mathrm{ps}$ at $\mathrm{T}=900 \mathrm{~K}, 6.6 \mathrm{ps}$ at $\mathrm{T}=600 \mathrm{~K}$ and $18.5 \mathrm{ps}$ at $300 \mathrm{~K}$. In the second, we had $6 \mathrm{ps}$ at $\mathrm{T}=300 \mathrm{~K}, 4.2 \mathrm{ps}$ at $\mathrm{T}=600 \mathrm{~K}, 12.3 \mathrm{ps}$ at $\mathrm{T}=900 \mathrm{~K}, 9.5 \mathrm{ps}$ at $\mathrm{T}=600 \mathrm{~K}$ and $16.4 \mathrm{ps}$ at $\mathrm{T}=300 \mathrm{~K}$. In both cases, at $\mathrm{T}=900 \mathrm{~K}$, further randomization is ensured by diffusion coefficients close to $2.5 \cdot 10^{-5} \mathrm{~cm}^{2} / \mathrm{s}$. The experimental glass transition temperature is $\mathrm{T}_{g}=445 \mathrm{~K}$ and the crystallization temperature is $\mathrm{T}_{c r}=560 \mathrm{~K}$. The melting temperature (no experiments available) is expected to be not larger than $\mathrm{T}=700 \mathrm{~K}$. In what follows, the results are presented as mean values of the 
two temporal averages on each distinct set of coordinates at $\mathrm{T}=300 \mathrm{~K}$.

\section{Structural properties: first-principles molecular dynamics results}

We exploit the partial pair correlation functions $\mathrm{g}_{\alpha \beta}(\mathrm{r})$ to obtain the total pair correlation function $\mathrm{g}_{T}(\mathrm{r})$, that can be extracted from a neutron scattering experiment, as follows:

$$
g_{T}(r)-1=\sum_{\alpha=1}^{3} \sum_{\beta=1}^{3} \frac{c_{\alpha} c_{\beta} b_{\alpha} b_{\beta}}{\langle b\rangle^{2}}\left[g_{\alpha \beta}(r)-1\right] .
$$

The quantities $c_{\alpha}$ and $b_{\alpha}$ are, respectively, the atomic fractions and coherent neutron scattering lengths of $\mathrm{Ga}(7.288 \mathrm{fm})$, Ge $(8.185 \mathrm{fm})$ and $\mathrm{Te}(5.800 \mathrm{fm})$. The mean coherent neutron scattering length $\langle b\rangle$ is defined as:

$$
\langle b\rangle=c_{G a} b_{G a}+c_{G e} b_{G e}+c_{T e} b_{T e} .
$$

The reciprocal space counterpart of the partial pair distribution functions are the Faber-Ziman (FZ) partial structure factors. From the standpoint of modelling, one method to obtain these quantities (alternative to direct calculation in reciprocal space), consists in Fourier transforming the $\mathrm{g}_{\alpha \beta}$ pair correlation functions

$$
S_{\alpha \beta}^{F Z}(k)-1=\frac{4 \pi \rho_{0}}{k} \int_{0}^{\infty} r\left[g_{\alpha \beta}(r)-1\right] \sin (k r) d r .
$$

Accordingly, the total neutron structure factor $\mathrm{S}_{T}(\mathrm{k})$ is a weighted sum of these partial quantities

$$
S_{T}(k)-1=\sum_{\alpha=1}^{3} \sum_{\beta=1}^{3} \frac{c_{\alpha} c_{\beta} b_{\alpha} b_{\beta}}{\langle b\rangle^{2}}\left[S_{\alpha \beta}^{F Z}(k)-1\right] .
$$

Our results in reciprocal space were obtained via the procedure outlined above by Fourier integration of the corresponding pair correlation functions.

In figure 1, we report the total neutron structure factor for our GGT system along with the experimental counterpart for the closest concentration available $\left(\mathrm{Ga}_{11} \mathrm{Ge}_{11} \mathrm{Te}_{78}\right)$ [6]. We find an excellent agreement with the measurements over the entire range of values in reciprocal space, both in terms of peaks positions and intensities. Our calculations are able to reproduce the little bump reminiscent of a FSDP (first sharp diffraction peak) feature around $1 \AA^{-1}$, indicative of a limited degree of intermediate range order that establishes in the network.

To seek the origins of this contribution, we report in figure 2, all calculated partial structure factors. No features are discernible around $1 \AA^{-1}$ in the case of the Te-Te and Ga-Te Faber-Ziman partial structure

90 factors. Peaks visible at values of $k$ smaller than $1 \AA^{-1}$ cannot be ascribed to any realistic structural feature, being due to the limited size of the simulation box and a statistical sampling affected by the periodicity. At 


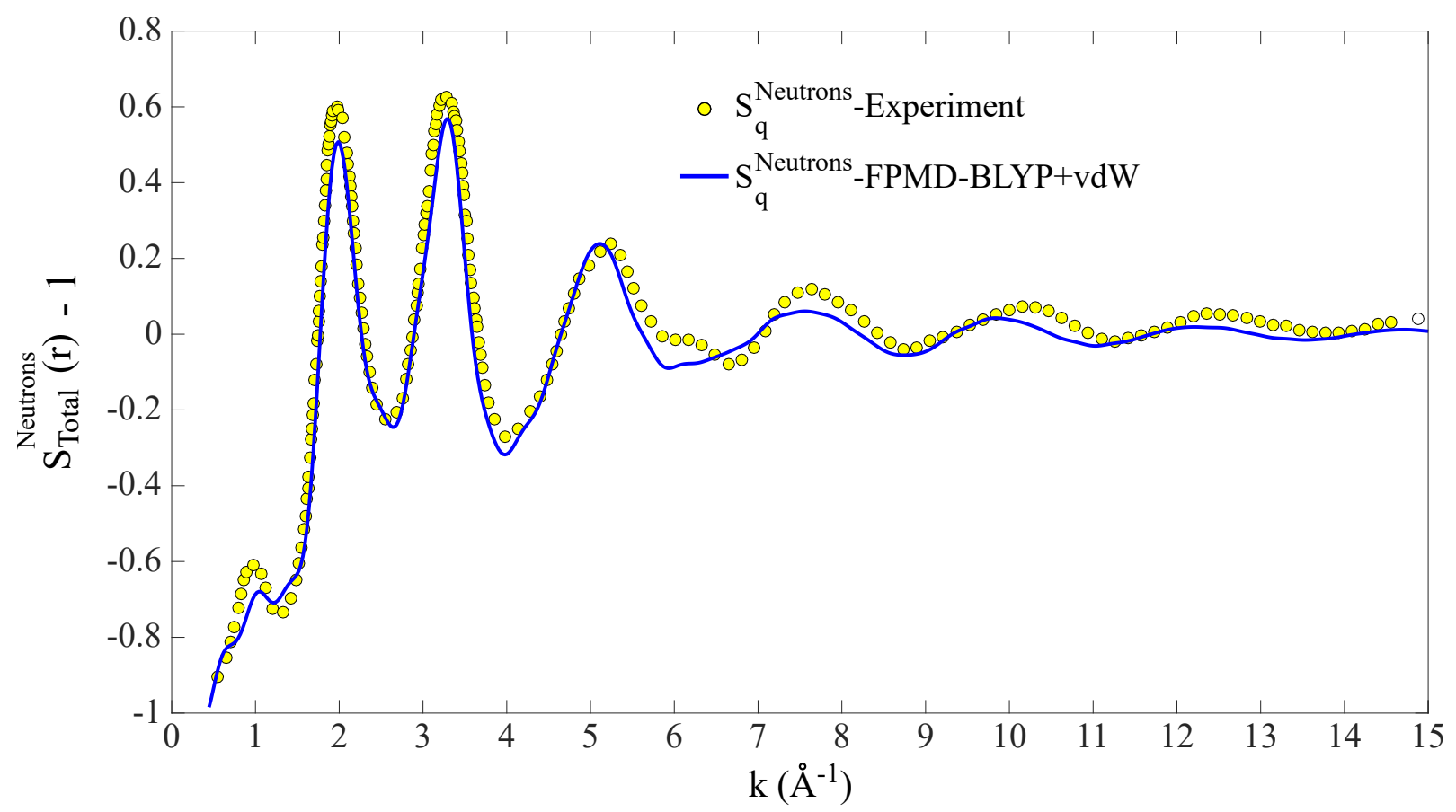

Figure 1: Calculated total neutron structure factor $\mathrm{S}(k)$ of glassy $\mathrm{Ga}_{10} \mathrm{Ge}_{15} \mathrm{Te} 75$ (blue solid line). The experimental data (circle symbols) are obtained from the work of Jóvári et al [6].

various levels of intensities, peaks are visible in the FSDP region for the Ge-Te, Ge-Ga, Ge-Ge and Ga-Ga partial structure factors. The most prominent is found in the Ge-Ga partial structure factor, followed by a smaller feature in the Ga-Ga one. Ge-Te correlations pertaining to intermediate range order are quite small, while in the case of Ge-Ge correlations, the FSDP-like peak is displaced to larger values $\left(1.5 \AA^{-1}\right)$ as to indicate the impact of a set of comparatively smaller distances. It should be noted that this rationale can barely be substantiated by the shape of $\mathrm{S}_{T}(\mathrm{k})$ around the FSDP region, due to the small concentration of Ge and Ga. Overall, the network topology of GGT does not exhibit an appreciable amount of intermediate range order, small detectable features being ascribed mostly to Ge correlations involving Ge, Ga and Te neighbors, the largest contribution being Ge-Ga. This is in line with the role played by Ge in several chalcogenide glasses, for which intermediate range order correlations are inevitably due to Ge atoms forming (mostly) tetrahedral units, edge-sharing or corner-sharing connected in space [16]. In this respect, Ga behaves differently, its impact on extended correlation being much less important. Among the three species, Te is the least prone to promote intermediate range order.

We calculated the partial pair correlation functions and compared them to those given by Voleská et al. [7]. These were obtained by FPMD starting from an initial structure generated via a Reverse Monte Carlo approach at a slightly different concentration $\left(\mathrm{Ga}_{11} \mathrm{Ge}_{11} \mathrm{Te}_{78}\right)$. We report these results in figure 3 . The two 

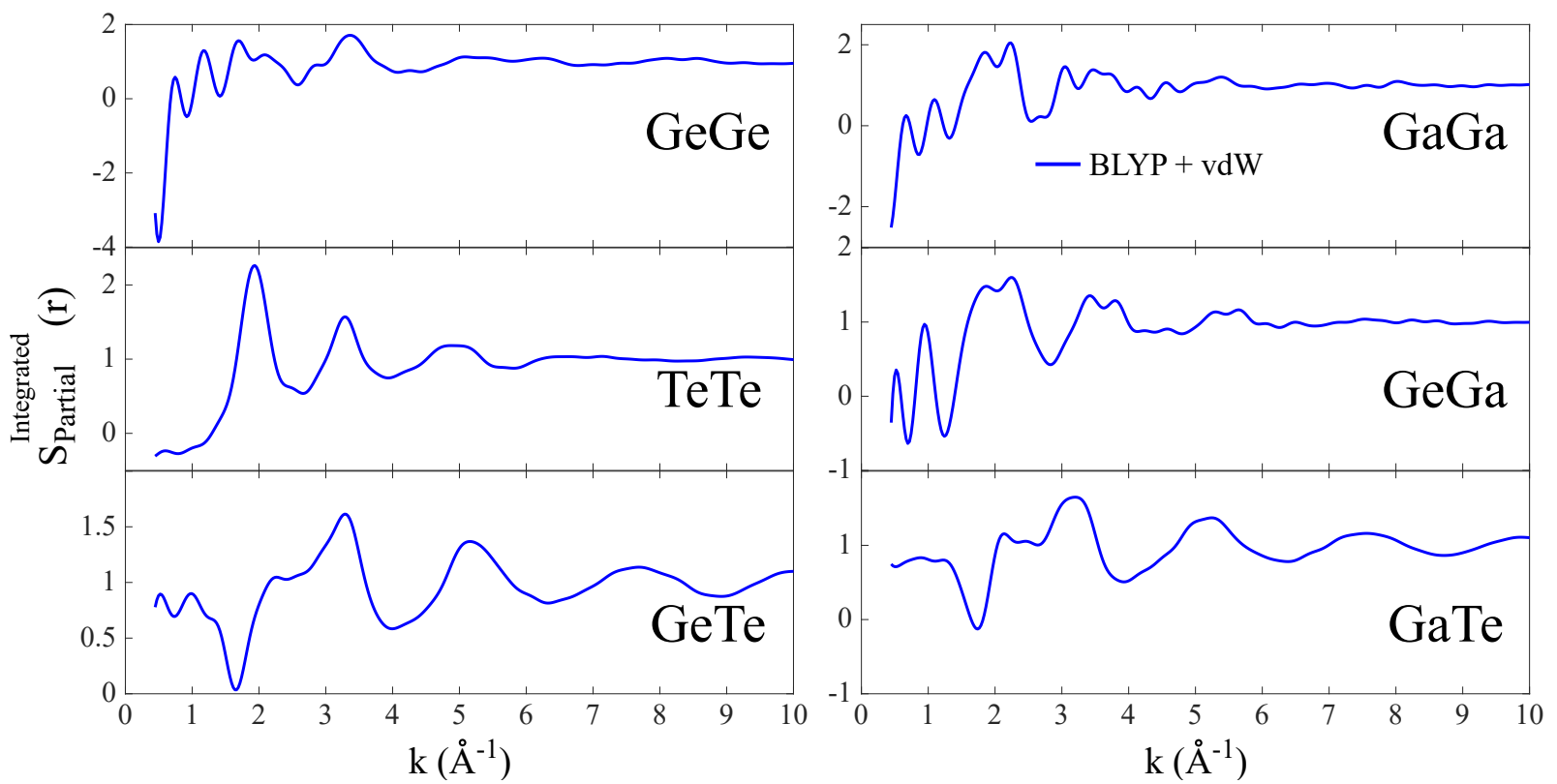

Figure 2: Faber-Ziman partial structure factors $\mathrm{S}_{i j}^{F Z}(\mathrm{i}, \mathrm{j}=\mathrm{Ge}, \mathrm{Ga}, \mathrm{Te})$ calculated within the FPMD-BLYP method including the vdW interactions scheme of the DFT-D2 method [21].

sets of Ge-Te and Ga-Te pair correlation functions are essentially identical, as if the differences between the two models had no effects on the spatial correlations. Nearest-neighbors interatomic distances are also very close (Ga-Te and Ge-Te distances are, respectively, $2.67 \AA$ and $2.59 \AA$ in our calculations against 2.63 $\AA$ and $2.65 \AA$ in Ref. [7]). Focusing on the $\mathrm{g}_{\alpha \beta}(\mathrm{r})$ associated with the homopolar connections Ga-Ga and Ge-Ge, we find a larger number of Ge-Ge homopolar bonds than Ga-Ga ones. The opposite occurs when using RMC-FPMD-PBE, resulting in $\mathrm{g}_{G a G a}(\mathrm{r})$ and $\mathrm{g}_{G e G e}(\mathrm{r})$ with broader and less intense second peaks, proving that the second shell of neighbors for these species is less structured. The most striking differences between FPMD-BLYP and RMC-FPMD-PBE are clearly visible for Ge-Ga correlations. In addition to a smaller first peak (smaller impact of Ge-Ga bonds), we found a fairly regular second large peak, definitely reducing in intensity for distances larger than $4 \AA$. This differs from the prediction of RMC-FPMD-PBE, for which Ge-Ga second neighbor distances lie at values higher than 4 A. Finally, differences between the two approaches are less severe in the pair correlation function Te-Te, with very close peaks at short distances and more pronounced minima in the present case.

Figure 3 contains also information on Ge-Ge, Ge-Te, Te-Te correlations by comparing our GGT model to glassy $\mathrm{GeTe}_{4}$, obtained within the same theoretical framework. Very close profiles are found in $\mathrm{g}_{G e T e}$ (a very intense first peak being noticeable), reflecting two networks sharing the same predominance of heteropolar 
bonding with structural units built around Ge. In the case of $\mathrm{g}_{G e G e}$, homopolar bonding is remarkably similar, as shown by the intensities of the first peak. The regular shape of the second peak in glassy $\mathrm{GeTe}_{4}$ is modified in glassy GGT due to the presence of a third species (Ga). The pair correlation functions $\mathrm{g}_{T e T e}$ are also quite close, with distinct shells of neighbors having pronounced maxima and minima within the range of first and second neighbor distances.
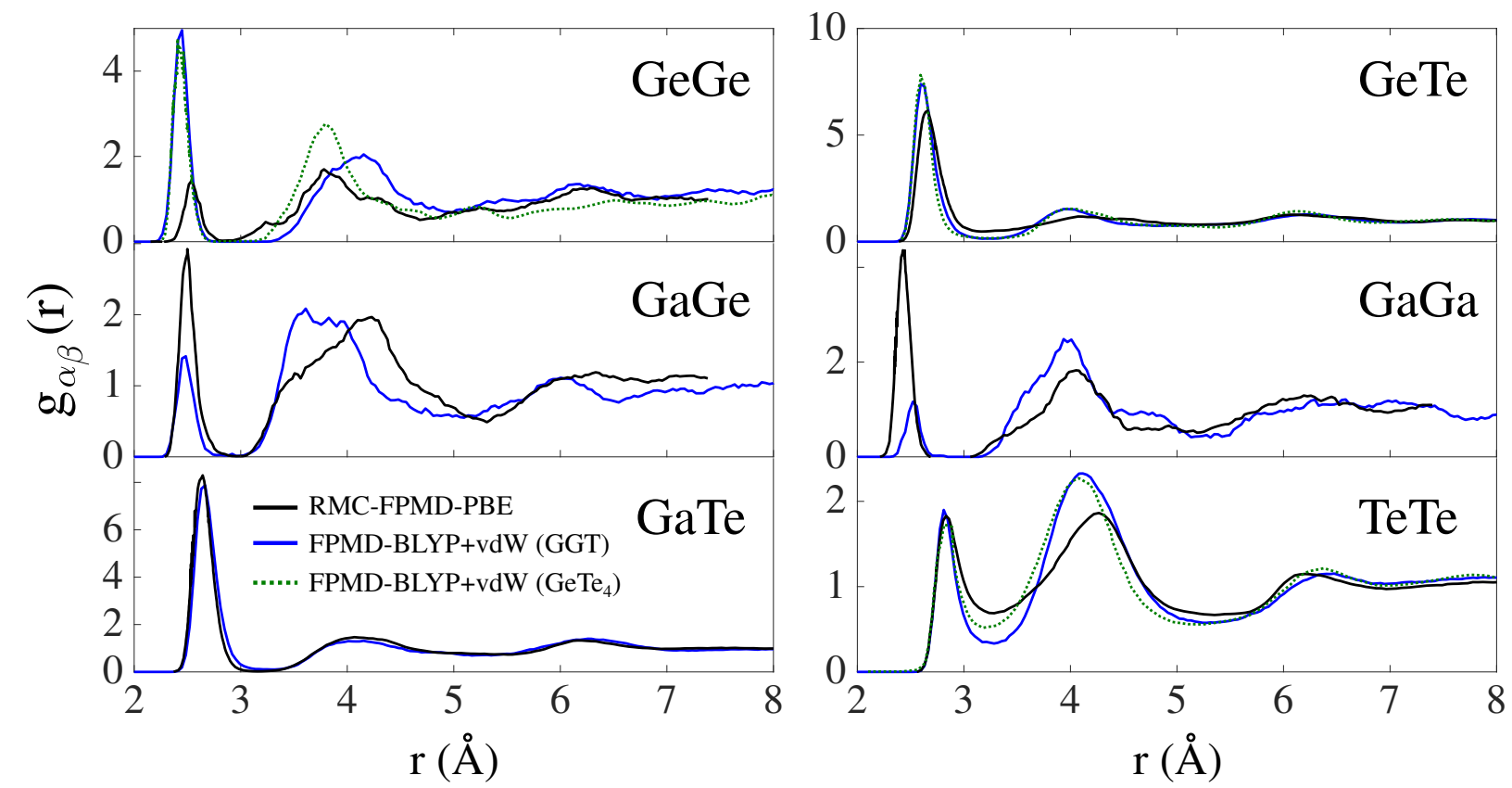

Figure 3: Partial pair correlation functions $\mathrm{g}_{\alpha \beta}(\alpha, \beta=\mathrm{Ge}, \mathrm{Ga}, \mathrm{Te})$ for the GGT system. Our FPMD-BLYP+vdW calculations:

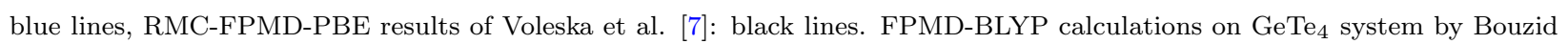
et al. [2]: green dotted lines

It is also of interest to ascertain the impact of the van der Waals contributions at the structural level. This can be done by including in the comparison the pair correlation functions calculated for glassy $\mathrm{GeTe}_{4}$ at the BLYP level with no vdW corrections. As shown in figure 4, the vdW part enhances the separation between shells of neighbors (Te-Te case) and the intensity of the first peak, related to homopolar bonds (Ge case). Within the same context, we have reported in figure 5 an additional comparison between three sets of Ge-Ge, Ge-Te and Te-Te pair correlation functions. We focus on glassy $\mathrm{GeTe}_{4}$ and glassy GGT via the RMC-FPMD-PBE approach (both without inclusion of vdW corrections, Ref. [7]) as well as our results that account for vdW corrections. As a self-explanatory example, we note that in $\mathrm{g}_{T e T e}$, the patterns found for glassy $\mathrm{GeTe}_{4}$ and glassy GGT (RMC-FPMD-PBE, no vdW) are fairly close and differ distinctly from our GGT, vdW inclusive data. A similar behavior is found in $\mathrm{g}_{G e G e}$.

Based on these pieces of evidence, it appears that a large extent of the differences between our GGT 
model and the one of Ref. [7] for $\mathrm{g}_{G e G e}$ and $\mathrm{g}_{T e T e}$ can be traced back to the account of van der Waals interactions.
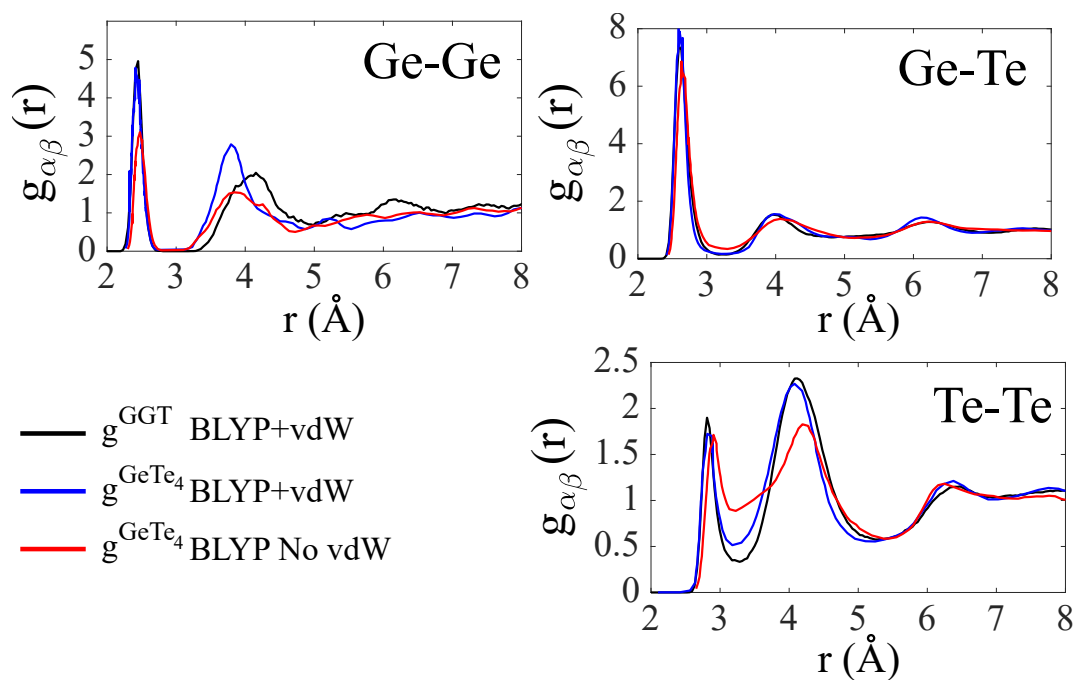

Figure 4: Atomic pair correlation functions for GeGe, GeTe and TeTe interactions pairs. The results of the present work for the GGT system (including vdW correction) are highlighted in black solid lines while the results obtained by Bouzid et al. [2] for $\mathrm{GeTe}_{4}$ are reported in two situations: including (blue lines) or not (red solid lines) vdW interactions
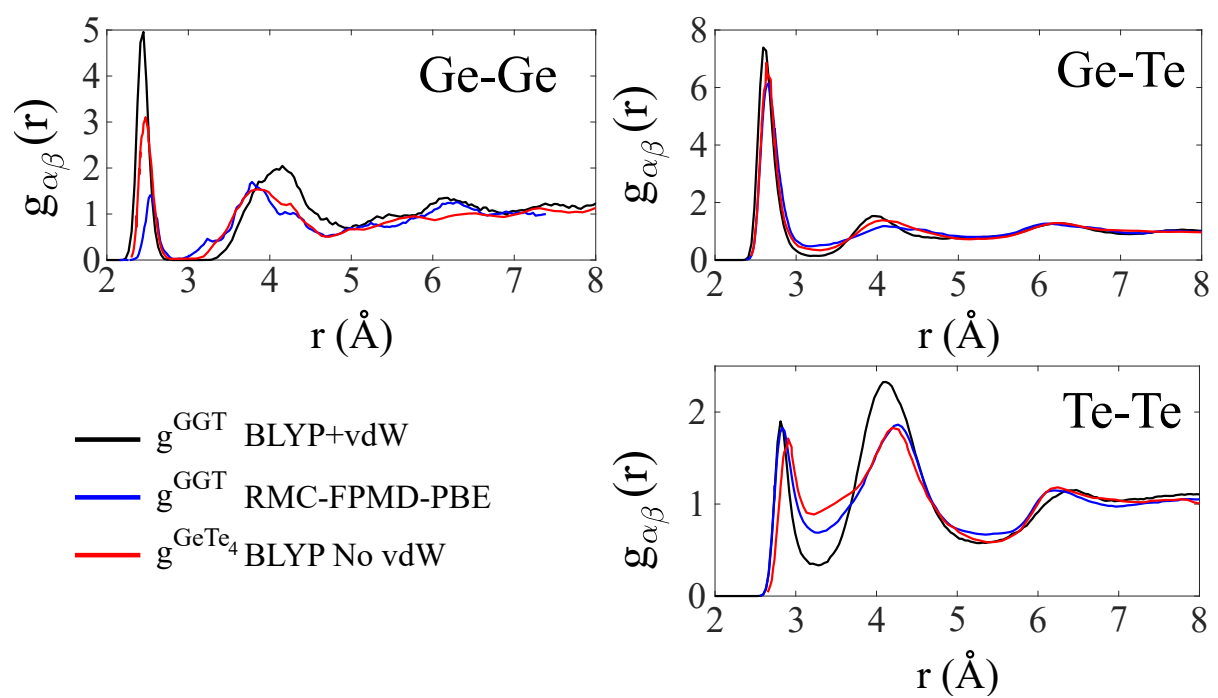

Figure 5: Atomic pair correlation functions for GeGe, GeTe and TeTe interaction pairs. The results of the present work for the GGT system (including vdW correction) are highlighted in black solid lines while the results obtained by Voleska et al. [7] for the $\mathrm{Ga}_{11} \mathrm{Ge}_{11} \mathrm{Te}_{78}$ correspond to the blue solid lines (no vdW correction). Results of Bouzid et al. [2] for the GeTe $\mathrm{T}_{4}$ glassy chalcogenide with no vdW interactions are given in red solid lines 


\section{B - Coordination numbers and analysis of local environment}

. We analyze the local order within the system through the evaluation of the corresponding coordination numbers. We use the set of experimental data provided by Jóvári et al. [6] and the RMC-FPMD-PBE results of Voleská et al. [7] in comparison with our findings. We report these data in a specific table (Table I). The partial coordination numbers are obtained by integration of the pair correlation function up to the first minimum. Having defined $c_{\alpha}$ as the concentration of each species, the average coordination number $<\mathrm{N}>$ is $<\mathrm{N}>=c_{G e} n_{G e}+c_{G a} n_{G a}+c_{T e} n_{T e}$, where the coordination numbers for each species are obtained by summing up the corresponding partial ones.

\begin{tabular}{lccc}
\hline \hline & Experiment & FPMD-BLYP+vdW & RMC-FPMD-PBE \\
\hline $\mathrm{r}_{\mathrm{GeGe}}$ & - & $2.45 \AA$ & $2.53 \AA$ \\
$\mathrm{r}_{\mathrm{GeGa}}$ & - & $2.49 \AA$ & $2.49 \AA$ \\
$\mathrm{r}_{\mathrm{GeTe}}$ & $2.60 \AA$ & $2.59 \AA$ & $2.65 \AA$ \\
$\mathrm{r}_{\mathrm{GaGa}}$ & - & $2.52 \AA$ & $2.43 \AA$ \\
$\mathrm{r}_{\mathrm{GaTe}}$ & $2.60 \AA$ & $2.67 \AA$ & $2.63 \AA$ \\
$\mathrm{r}_{\mathrm{TeTe}}$ & $2.80 \AA$ & $2.81 \AA$ & $2.83 \AA$ \\
\hline $\mathrm{N}_{\mathrm{GeGe}}$ & - & 0.24 & 0.07 \\
$\mathrm{~N}_{\mathrm{GeGa}}$ & - & 0.06 & 0.14 \\
$\mathrm{~N}_{\mathrm{GeTe}}$ & 3.97 & 3.44 & 3.55 \\
$\mathrm{~N}_{\mathrm{GaGa}}$ & - & 0.02 & 0.17 \\
$\mathrm{~N}_{\mathrm{GaTe}}$ & 3.00 & 3.78 & 3.77 \\
$\mathrm{~N}_{\mathrm{TeTe}}$ & 1.36 & 1.00 & 1.52 \\
\hline $\mathrm{n}_{\mathrm{Ge}}$ & - & 3.73 & - \\
$\mathrm{n}_{\mathrm{Ga}}$ & - & 3.89 & - \\
$\mathrm{n}_{\mathrm{Te}}$ & 2.36 & 2.19 & - \\
$\langle\mathrm{N}\rangle$ & 2.61 & 2.59 & - \\
\hline \hline
\end{tabular}

Table I: Bonds properties (interatomic distances) and the corresponding coordination numbers for the GGT system calculated with the FPMD-BLYP+vdW method (central column) compared to the experimental results of Jóvári et al. (leftmost column) [6] and the RMC-FPMD-PBE of Voleska et al. (rightmost column) [7]

In terms of the bond distances, the three sets of results agree to a good extent, indicating that the gross topology of the network is well reproduced. Regarding the coordination numbers, two main features are worth pointing out. First, Ga and Ge environments are consistent with a predominant fourfold coordination, Te atoms being arranged either in units around Ge or Ga or in homopolar connections. This is in contrast with the predictions extracted from experiments, leading to an average number of 3 Te atoms coordinating Ga 
atoms. Second, the present approach favors a higher number of Ge-Ge homopolar bonds, with a few Ga-Ga ones. The opposite occurs in the RMC-FPMD-PBE case. This observation is consistent with the shapes of the pair correlation functions.

\begin{tabular}{|c|c|c|c|c|c|c|c|}
\hline & & \multicolumn{2}{|c|}{ proportion $\mathrm{p}_{\alpha}(\mathrm{l})[\%]$} & & & \multicolumn{2}{|c|}{ proportion $\mathrm{p}_{\alpha}(1)[\%]$} \\
\hline & & FPMD-BLYP+vdW & RMC-FPMD-PBE & & & FPMD-BLYP+vdW & RMC-FPMD-PBE \\
\hline \multicolumn{4}{|l|}{ Ge atom } & \multicolumn{4}{|l|}{ Te atom } \\
\hline$l=1$ & $\mathrm{Te}$ & $<0.1$ & - & \multirow{4}{*}{$l=2$} & $\mathrm{Ge}$ & 0.99 & $<1$ \\
\hline$l=2$ & $\begin{array}{l}\mathrm{GeTe} \\
\mathrm{Te}_{2}\end{array}$ & $\begin{array}{l}<0.1 \\
1.07\end{array}$ & $<1$ & & $\begin{array}{l}\mathrm{Ga} \\
\mathrm{Te}\end{array}$ & $\begin{array}{l}0.26 \\
1.79\end{array}$ & \\
\hline$l=3$ & $\begin{array}{l}\mathrm{Te}_{3} \\
\mathrm{GeTe}_{2}\end{array}$ & $\begin{array}{c}22.02 \\
2.47\end{array}$ & $\begin{array}{c}23 \\
-\end{array}$ & & $\begin{array}{l}\mathrm{Ge}_{2} \\
\mathrm{Ga}_{2}\end{array}$ & $\begin{array}{l}7.18 \\
2.90\end{array}$ & $\begin{array}{l}1.9 \\
1.3\end{array}$ \\
\hline \multirow[t]{2}{*}{$l=4$} & $\begin{array}{l}\mathrm{Te}_{4} \\
\mathrm{GaTe}_{3} \\
\mathrm{GeTe}_{3} \\
\mathrm{Ge}_{2} \mathrm{Te}_{2}\end{array}$ & $\begin{array}{c}51.57 \\
5.15 \\
13.5 \\
3.48\end{array}$ & $\begin{array}{c}54.5 \\
10.5 \\
5.0 \\
3.48\end{array}$ & & $\begin{array}{l}\mathrm{Te}_{2} \\
\mathrm{GeGa} \\
\mathrm{GeTe} \\
\mathrm{GaTe}\end{array}$ & $\begin{array}{l}17.05 \\
10.52 \\
23.51 \\
14.28\end{array}$ & $\begin{array}{c}16.0 \\
6.6 \\
12.6 \\
12.3\end{array}$ \\
\hline & $\mathrm{GeGaTe}_{2}$ & 0.70 & 1.7 & \multirow[t]{9}{*}{$l=3$} & $\mathrm{Ge}_{3}$ & 0.44 & - \\
\hline \multirow[t]{4}{*}{$l=5$} & $\begin{array}{l}\mathrm{Te}_{5} \\
\mathrm{GeTe}\end{array}$ & $\begin{array}{l}<0.1 \\
<01\end{array}$ & 3.3 & & $\mathrm{Ga}_{3}$ & 0.52 & - \\
\hline & $\mathrm{GeTe}_{4}$ & $<0.1$ & - & & $\mathrm{Te}_{3}$ & 1.66 & 9.3 \\
\hline & $\mathrm{GeGaTe}_{3}$ & $<0.1$ & - & & $\mathrm{GeGa}_{2}$ & 1.71 & - \\
\hline & $\mathrm{Ge}_{2} \mathrm{Te}_{3}$ & - & - & & $\mathrm{Ge}_{2} \mathrm{Ga}$ & 2.07 & 2.2 \\
\hline \multicolumn{4}{|l|}{ Ga atom } & & $\mathrm{Ge}_{2} \mathrm{Te}$ & 2.51 & - \\
\hline \multicolumn{2}{|l|}{$l=0$} & 0.63 & - & & $\mathrm{GeTe}_{2}$ & 2.34 & 9.0 \\
\hline \multirow{2}{*}{$l=1$} & $\mathrm{Te}$ & 0.96 & \multirow[t]{2}{*}{-} & & $\mathrm{GaTe}_{2}$ & 3.04 & 9.7 \\
\hline & $\mathrm{Ge}$ & $<0.1$ & & & $\begin{array}{l}\mathrm{Ga}_{2} \mathrm{Te} \\
\mathrm{GeGaTe}^{2}\end{array}$ & $\begin{array}{l}2.19 \\
4.70\end{array}$ & $\begin{array}{l}2.7 \\
6.4\end{array}$ \\
\hline \multirow[t]{2}{*}{$l=2$} & $\mathrm{Te}_{2}$ & 0.59 & \multirow[t]{2}{*}{-} & \multirow{7}{*}{$l=4$} & $\mathrm{Te}_{4}$ & $<0.1$ & 1.6 \\
\hline & GeTe & $<0.1$ & & & $\mathrm{GeTe}_{3}$ & $<0.1$ & 1.2 \\
\hline \multirow[t]{3}{*}{$l=3$} & & 4.65 & \multirow[t]{3}{*}{$<1$} & & $\mathrm{Ge}_{2} \mathrm{Te}_{2}$ & $<0.1$ & - \\
\hline & $\mathrm{GeTe}_{2}$ & $<0.1$ & & & $\mathrm{GaTe}_{3}$ & $<0.1$ & - \\
\hline & $\mathrm{GaTe}_{2}$ & 0.14 & & & $\mathrm{Ga}_{2} \mathrm{Te}_{2}$ & 0.10 & - \\
\hline \multirow[t]{3}{*}{$l=4$} & $\mathrm{Te}_{4}$ & 82.33 & \multirow{3}{*}{$\begin{array}{l}69.7 \\
13.3 \\
16.1\end{array}$} & & $\mathrm{GeGaTe}_{2}$ & $<0.1$ & 1.1 \\
\hline & $\mathrm{GeTe}_{3}$ & 8.62 & & & $\mathrm{GeGa}_{2} \mathrm{Te}$ & $<0.1$ & - \\
\hline & $\mathrm{GaTe}_{3}$ & 1.73 & & \multirow{3}{*}{$\begin{array}{c}\text { Homopolar } \\
\text { bonds }\end{array}$} & & & \\
\hline \multirow[t]{2}{*}{$l=5$} & $\mathrm{Te}_{5}$ & $<0.1$ & - & & Ge atom & Ga atom & Te atom \\
\hline & $\begin{array}{l}\mathrm{GeTe}_{4} \\
\mathrm{GaTe}_{4}\end{array}$ & $\begin{array}{l}<0.1 \\
0.21\end{array}$ & & & $20.30 \%$ & $2.08 \%$ & $73.47 \%$ \\
\hline
\end{tabular}

Table II: Percentage population $\mathrm{p}_{\alpha}(\mathrm{l})$ of the different coordination units in glassy GGT for the specie $\alpha$ (Ga, Ge or Te). These quantities have been calculated including neighbors separated by a cutoff corresponding to the first minimum in the partial pair correlation functions. The atomic species are indicated in bold and the coordination numbers are classified with respect to the number $(l)$ of neighbors and their type. The results of the present work are reported in the first column and those of Voleska et al. [7] in the second column. In the bottom right part, the quantification of homopolar bonds for each species is extracted from our FPMD-BLYP calculations. We provide the percentage number of Ge, Ga and Te involved in at least one homopolar bond

In order to go further into the local coordination analysis, we show in Table II a detailed table of coordination numbers in which each element is considered separately. The contributions are split according to the species involved in each particular coordination unit. We compare our results with those obtained by Voleská et al. [7] (RMC-FPMD-PBE approach). The two sets of data agree on the nature of the Ge coor- 
dination, mostly fourfold and threefold, these latter connections being indicative of departures from ordered heterogeneous bonding. When considering the fourfold Ge coordinated atoms, most of them are bonded to Te atoms (more than 50 \%). At first sight, both approaches also agree on the predominant fourfold character of the Ga connections.

However, the weights of the specific units are different, since RMC-FPMD-PBE favors Ga-Ga bonding within the $\mathrm{Ga}_{-} \mathrm{GaTe}_{3}$ coordination $(16.1 \%)$. This is at the very origin of the Ga-Ga intense peak in the corresponding pair correlation function. Also, the present results are indicative of a majority of GaTe $\mathrm{T}_{4}$ units, revealing the strong tendency of the FPMD-BLYP scheme to minimize deviations from the fourfold coordination in the case of $\mathrm{Ga}$, together with a little number of homopolar bonds. In the case of Te, the coordination is mainly twofold, as expected in view of simple chemical considerations on the valence character of this element. However, there are a variety of different coordination subsets, all the possible twofold combinations being represented, namely Ge-Te-Ge, Ge-Te-Te, Te-Te-Te, Ga-Te-Ga, Ga-Te-Te and Ga-Te-Ge. Sizeable fractions of such units are found in a network in which tetrahedral (or defective octahedral) units are cross-linked through connections of Te atoms that can also form chains of various lengths [16, 27].

\section{C-Analysis of the network topology}

- To identify the nature of the fourfold coordination for Ge and Ga atoms, we used a local order parameter, introduced by Chau [28] and Errington [29] and recently employed by Bouzid et al. [2] to characterize the tetrahedral/octahedral local atomic environments in glassy $\mathrm{GeTe}_{4}$. It is defined as:

$$
q=1-\frac{3}{8} \sum_{k>i}\left[\frac{1}{3}+\cos \left(\theta_{i j k}\right)\right]^{2}
$$

In this expression, $\theta_{i j k}$ is the angle formed between the central atom $j$ and its neighboring atoms $i$ and $k$. The values of this specific order parameter vary from 0 (perfect sixfold octahedral sites) to 1 (ideal tetrahedral network), the intermediate values indicating various defective octahedral and tetrahedral configurations [5]. The distribution of occurrences is reported in figure 6-(a) for both the Ge- and Ga-coordination. We observe that the Ge glass network is mostly of tetrahedral nature as confirmed by the main peak close to $\mathrm{q}=1$. The main contributions come from the three- and mostly four-fold coordination in line with our analysis of the coordination numbers (Table II). However, there is also a very small fraction of defective octahedra as exemplified by the broad feature obtained in between $q=0.6$ and $q=0.8$. A similar analysis can be applied to the Ga coordination environment. However, in this case, the profile of the local order parameter is essentially flat in between 0 and 0.7 (vanishing octahedral contributions) and the main peak indicative of tetrahedral coordination has a smaller weight of threefold units than the Ge-counterpart. The tetrahedral character of both the four-fold Ge and Ga subnetworks is confirmed by visual inspection of the snapshot given in figure 6-(c), where in addition one can spot the high amount of homopolar Te-Te bonds (figure 6-(b)). 
(a)

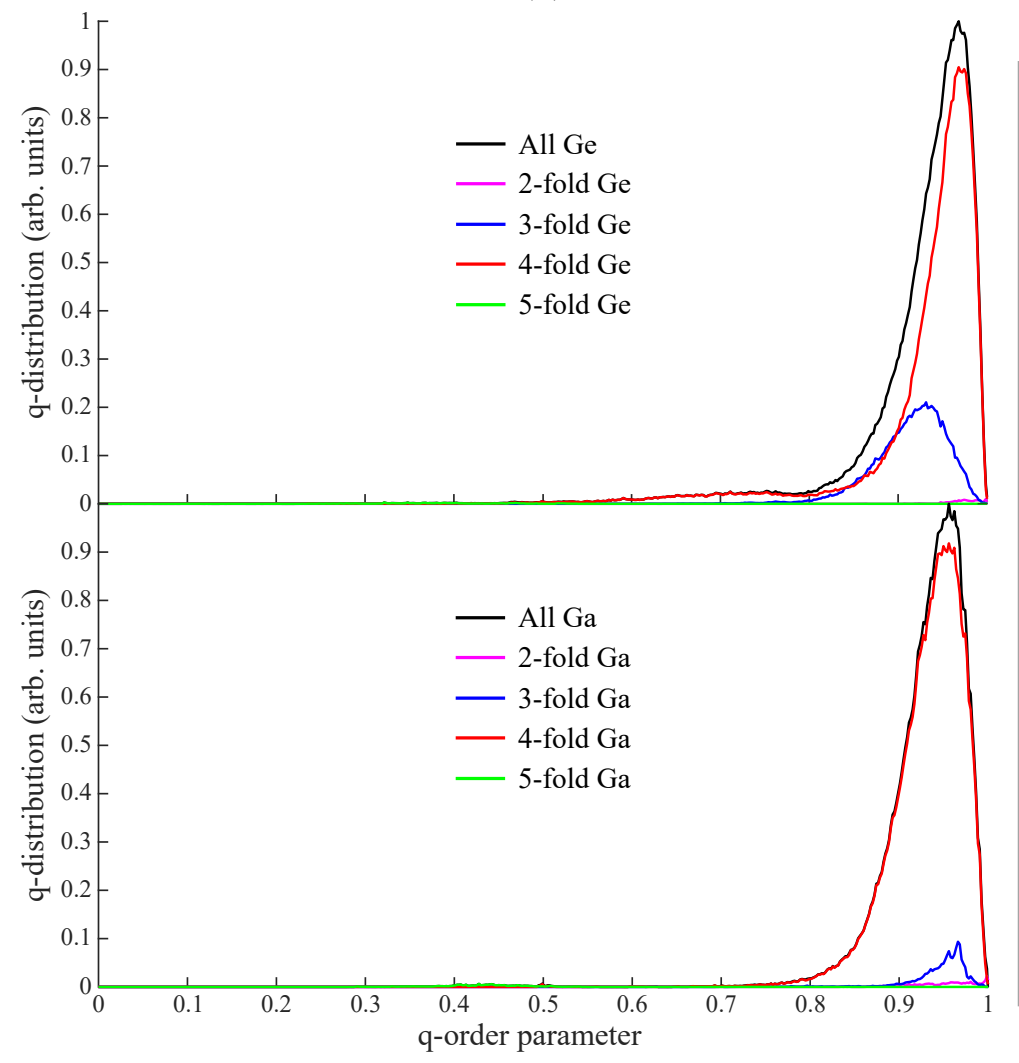

(b)

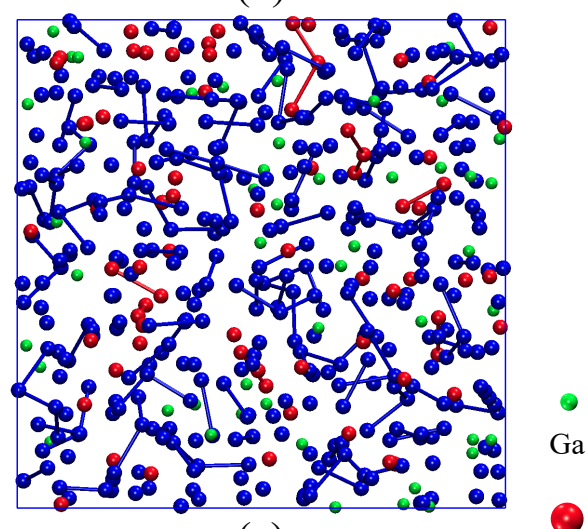

(c)

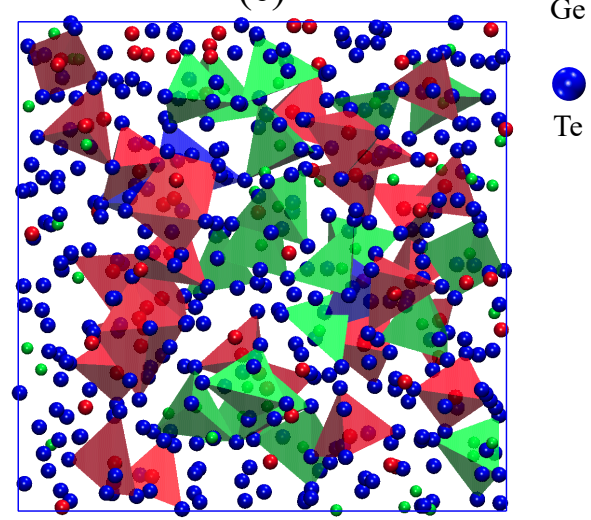

Figure 6: (a) Distribution of the local atomic coordination motifs (for Ga and Ge atoms) as a function of the order parameter $q$. (b) Snapshot of the simulation cell considered in our FPMD calculations of the GGT system. We highlight the predominance of the Te-Te homopolar bonds (blue) and the lower amount of Ge-Ge (green) and Ga-Ga (red) bonds. (c) Visualization of the tetrahedral arrangements characteristic of the GGT structure for both Ge and Ga atoms.

As an additional piece of information, we provide in figure 7, the angular distribution functions for triads Te-Te-Te, Ge-Te-Ge and Ga-Te-Ga. Te atoms in chains are arranged in configurations compatible with angles distributed around $90^{\circ}$ and $165^{\circ}$, while for $\mathrm{Ga}$ and $\mathrm{Ge}$ centered angular correlations, there is a clear evidence of a tetrahedral arrangement with maxima around $110^{\circ}$. 


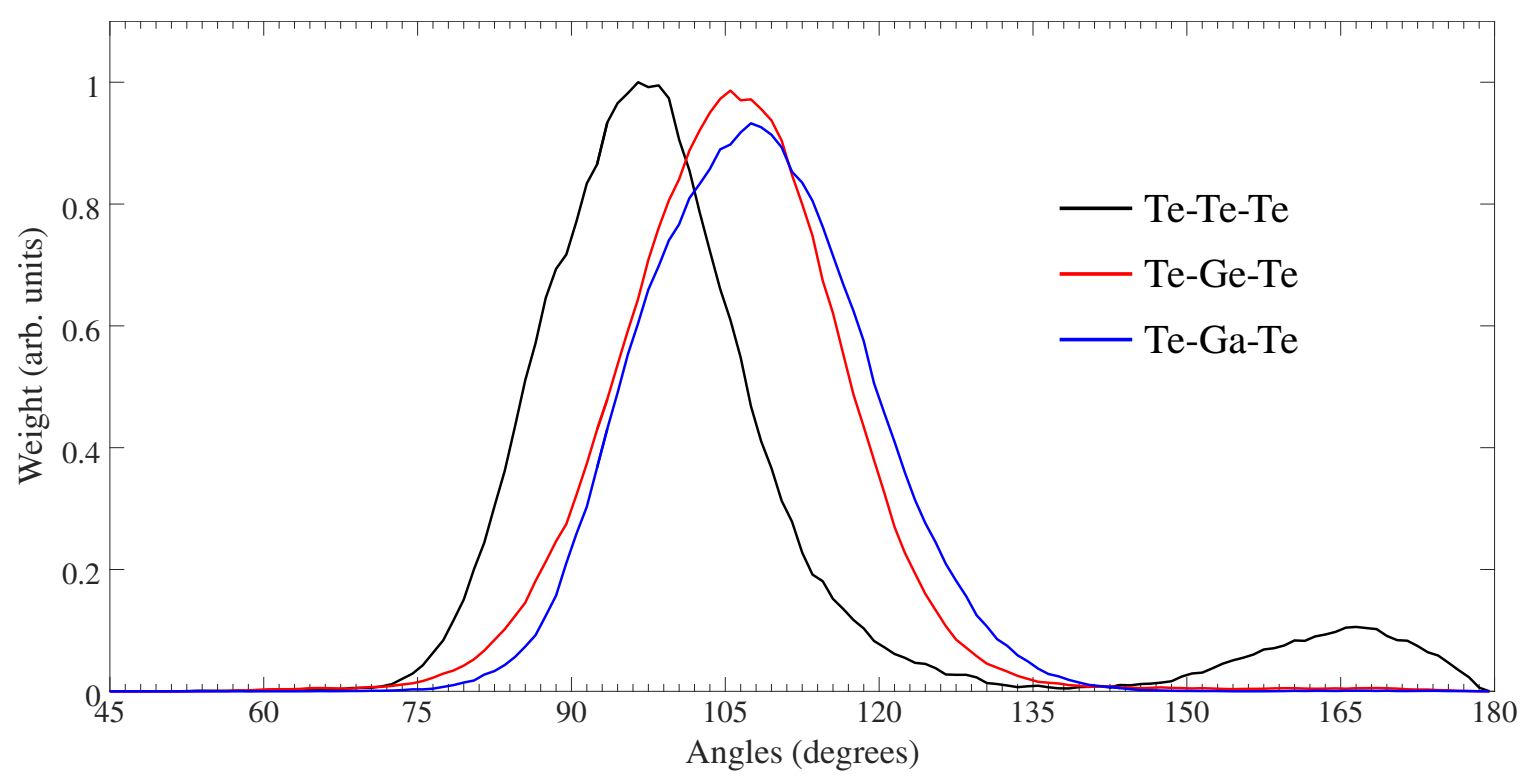

Figure 7: (Color online) Bond angle distribution (BAD) of the triplets Te-Te-Te (black solid line), Te-Ge-Te (red line) and TeGa-Te (blue line) in the atomic structure of glassy Ga10 Ge $15 \mathrm{Te}_{75}$ (GGT). The three data sets are obtained through averaging over two first-principles molecular dynamics trajectories at room temperature (details of the systems in the computational methods section)

\section{Conclusion}

We have obtained a structural description of glassy $\mathrm{Ga}_{10} \mathrm{Ge}_{15} \mathrm{Te}_{75}$ by relying on first-principles molecular dynamics. In the past, a system very close in concentration was considered with a similar approach, by combining experimental information (by means of reverse Monte Carlo scheme) [7] to FPMD. While those results remain a valuable reference to compare with, we demonstrate in this work that first-principles molecular dynamics is intrinsically capable of describing realistically a disordered ternary system without the need of any input from experimental results. Comparison with experimental structural data (neutron diffraction) turns out to be excellent, thereby conferring a very good level of legitimacy to our approach. Glassy $\mathrm{Ga}_{10} \mathrm{Ge}_{15} \mathrm{Te}_{75}$ is a predominantly tetrahedral network, in which $\mathrm{Ga}$ and Ge atoms are largely fourfold coordinated to Te atoms. In addition to the expected formation of Te-Te homopolar bonds, Ge atoms are also found in Ge-Ge configurations, while Ga-Ga configurations are less abundant. Dispersion forces are by no means negligible in determining the network structure, in line with previous results obtained for glassy $\mathrm{GeTe}_{4}[2]$. 


\section{Acknowledgments}

We acknowledge the support of the French Agence Nationale de la Recherche (ANR) through the project IrTeglass (ANR-14-CE07-0013). We are grateful to the Direction Informatique (Pôle HPC) of the University of Strasbourg for backing this work by providing scientific support and access to computing resources. Part

\section{References}

\section{Bibliography}

[1] S. Danto, P. Houizot, C. Boussard-Pledel, X.-H. Zhang, F. Smektala, J. Lucas, A family of far-infraredtransmitting glasses in the Ga-Ge-Te system for space applications, Advanced Functional Materials

[2] A. Bouzid, C. Massobrio, M. Boero, G. Ori, K. Sykina, E. Furet, Role of the van der Waals interactions and impact of the exchange-correlation functional in determining the structure of glassy $\mathrm{GeTe}_{4}$, Physical Review B 92 (13) (2015) 134208.

[3] P. Lucas, C. Boussard-Pledel, A. Wilhelm, S. Danto, X.-H. Zhang, P. Houizot, S. Maurugeon, C. Conseil,

[6] P. Jóvári, I. Kaban, B. Bureau, A. Wilhelm, P. Lucas, B. Beuneu, D. A. Zajac, Structure of Te-rich Te-Ge-X (X= I, Se, Ga) glasses, Journal of Physics: Condensed Matter 22 (40) (2010) 404207.

[7] I. Voleská, J. Akola, P. Jóvári, J. Gutwirth, T. Wágner, T. Vasileiadis, S. Yannopoulos, R. Jones, Structure, electronic, and vibrational properties of glassy $\mathrm{Ga}_{11} \mathrm{Ge}_{11} \mathrm{Te}_{78}$ : Experimentally constrained

[8] R. Car, M. Parrinello, Unified approach for molecular dynamics and density-functional theory, Physical review letters 55 (22) (1985) 2471.

[9] J. Hutter, M. Parrinello, D. Marx, P. Focher, M. Tuckerman, W. Andreoni, A. Curioni, E. Fois, U. Rothlisberger, P. Giannozzi, et al., Computer code cpmd, IBM Zurich Research Laboratory and MPI für 
[10] A. D. Becke, Density-functional exchange-energy approximation with correct asymptotic behavior, Physical review A 38 (6) (1988) 3098.

[11] C. Lee, W. Yang, R. G. Parr, Development of the Colle-Salvetti correlation-energy formula into a functional of the electron density, Physical review B 37 (2) (1988) 785.

[21] S. Grimme, Semiempirical GGA-type density functional constructed with a long-range dispersion correction, Journal of computational chemistry 27 (15) (2006) 1787-1799.

[22] P. Hohenberg, W. Kohn, Inhomogeneous electron gas, Physical review 136 (3B) (1964) B864. 
[23] W. Kohn, L. J. Sham, Self-consistent equations including exchange and correlation effects, Physical Review 140 (4A) (1965) A1133.

[24] S. Nosé, A unified formulation of the constant temperature molecular dynamics methods, The Journal of chemical physics 81 (1) (1984) 511-519.

[25] W. G. Hoover, Canonical dynamics: equilibrium phase-space distributions, Physical Review A 31 (3) (1985) 1695.

[26] P. E. Blöchl, M. Parrinello, Adiabaticity in first-principles molecular dynamics, Physical Review B 45 (16) (1992) 9413.

[27] K. Sykina, E. Furet, B. Bureau, S. Le Roux, C. Massobrio, Network connectivity and extended Se chains in the atomic structure of glassy $\mathrm{GeSe}_{4}$, Chemical Physics Letters 547 (2012) 30-34.

[28] P.-L. Chau, A. Hardwick, A new order parameter for tetrahedral configurations, Molecular Physics 93 (3) (1998) 511-518.

[29] J. R. Errington, P. G. Debenedetti, Relationship between structural order and the anomalies of liquid water, Nature 409 (6818) (2001) 318-321. 